\title{
HOMOGENEITY BY ISOTOPY
}

\author{
GENE G. GARZA
}

\begin{abstract}
Answered is a question asked by Daverman, Bull. Amer. Math. Soc. 84 (1978), 377-405. It is whether a 2-sphere $\Sigma$ is tame if each isotopy on $\Sigma$ extends to an isotopy of $E^{3}$ ?
\end{abstract}

In [2], Daverman asked several interesting questions concerning homogeneity. In particular, he asked if a 2-sphere $\Sigma$ is tame if each isotopy on $\Sigma$ extends to an isotopy of $E^{3}$ ? The answer is given by the following theorem. ${ }^{1}$

THEOREM. If $\Sigma$ is a 2-sphere which is homogeneous by isotopy, then $\Sigma$ is tame.

Proof. Parameterize some cell on $\Sigma$ by $I^{2}=[0,1] \times[0,1]$. Let $A$ be an arc which pierces $\Sigma$ at $(0,0)$ and is parameterized by a homeomorphism $\varphi$ : $[-1,1] \rightarrow A$ with $\varphi(0)=(0,0) \in I^{2}$. Let $H: E^{3} \times I \rightarrow E^{3}$ be the extension of an isotopy on $\Sigma$ which moves $\varphi(0)$ to $(1,0)$ by sliding the left side of the unit square over to the right side in such a manner that, at time $t,\left(0, t_{1}\right)$ is at $\left(t, t_{1}\right)$. Let $G$ be such an extension which moves the bottom of $I^{2}$ to the top. Having done this we see that $G(H(\varphi(0), I), I)$ is the unit square $I^{2}$ on $\Sigma$ and we need only to define

$$
g: G(H(\phi(0), I), I) \times[-1,1] \rightarrow G(H(A, I), I)
$$

by

$$
g\left(G\left(H\left(\varphi(0), t_{1}\right), t_{2}\right), t\right)=G\left(H\left(\phi(t), t_{1}\right) t_{2}\right)
$$

Now by Daverman's "singular regular neighborhoods" [1] we are done.

Note that by [1], this argument also holds for any $(n-1)$-sphere in $E^{n}$, $n \neq 4$.

ADDENDUM. This is the theorem to which Daverman was referring in [2, p. 387].

Received by the editors August 15, 1978.

AMS (MOS) subject classifications (1970). Primary 57A10, 57A45.

Key words and phrases. Homogeneous by isotopy, tame.

'This theorem is taken from the author's dissertation at the University of Georgia which was written under the direction of Professor James Cantrell. 


\section{BIBLIOGRAPHY}

1. R. J. Daverman, Singular regular neighborhoods and local flatness in codimension one, Proc. Amer. Math. Soc. 57 (1976), 357-362.

2. Embeddings of $(n-1)$-spheres in Euclidean n-space, Bull. Amer. Math. Soc. 84 (1978), 377-405.

Department of Mathematics, University of Grorgia, Athens, Grorgia 30602

Current address: Department of Mathematics, Wingate College, Wingate, North Carolina 28174 